\title{
Los Acuerdos de Paz de la Habana: una oportunidad para disminuir la pobreza y la exclusión rural en Sucre, Colombia
}

\section{The Peace Agreements of Habana: an opportunity to reduce poverty and rural exclusion in Sucre, Colombia}

\author{
Jaimes-Amorocho Hernando ${ }^{1}$ M.Sc; Lugo H Emily² M.Sc.
}

${ }^{1}$ Universidad Cooperativa de Colombia. Montería, Córdoba. ${ }^{2}$ Corporation Universitaria Antonio José de Sucre, Colombia.

\section{Keywords:}

Rural development; peace agreement; agricultural reform; Sucre;

Colombia.

\section{Abstract}

The rural regions occupy an extended part of the countries and they are characterized by their importance for the development and the social cohesion of the populations; which makes necessary the evaluation of policies and the context of these zones in rural territories like the department of Sucre. In this sense, the present document analyzes, through a tour of the country's agrarian regulations, the characteristics of the rural areas of the department and the proposal established in the peace agreements of Havana, to determine the opportunities that are Could present to improve the economic and social conditions in the department of Sucre.

\section{Palabras Clave:}

Desarrollo rural; acuerdo de paz; reforma agraria; Sucre;

Colombia.

\section{Resumen}

Las regiones rurales ocupan una gran extensión de los países y se caracterizan por su importancia para el desarrollo y la cohesión social de las poblaciones; lo que hace necesaria la evaluación de políticas y el contexto de estas zonas en territorios rurales como el Departamento de Sucre. En este sentido, el presente documento hace un análisis, a través de un recorrido por la normatividad agraria del país, de las características de las zonas rurales del Departamento y la propuesta establecida en los acuerdos de paz de la Habana, para determinar las oportunidades que se podrían presentar para mejorar las condiciones económicas y sociales en el Departamento de Sucre. 


\section{Introducción}

Los Acuerdos de Paz de la Habana: una oportunidad para disminuir la pobreza y la exclusión rural en Sucre.

"En materia de ordenamiento social de la propiedad, Colombia requiere una profunda transformación agraria que contenga, entre otros elementos, estrategias para reducir la generalizada informalidad que caracteriza al sector rural, la altísima concentración en la estructura de la propiedad agraria y la delicada situación de minifundio que ahoga a ciertas regiones del país." (DNP, 2015). Introducción

De acuerdo a información del Banco Mundial, el $70 \%$ los pobres del mundo (2017) viven en zonas rurales, dedicados a la práctica de actividades como la agricultura, la ganadería y la minería, que ocupan grandes extensiones de tierra, aportan el 32,1\% del valor agregado del PIB mundial y juegan un rol importante en la gestión de los recursos y en la economía de los países.

La dependencia de estas zonas, de sectores como el agropecuario, el crecimiento y reactivación de las actividad asociadas al sector, hacen que este sea considerado como uno de los factores que debería jalonar la reducción de los desequilibrios entre el campo y la ciudad y, por esa vía, reducir las diferencias regionales, generando de esta forma mejores condiciones de vida para sus habitantes (GAMARRA, 2009; ASHLEY y MAXWELL, 2001).

No obstante, el panorama en Colombia para el territorio rural no es promisorio, en contraste con la riqueza cultural y natural existente en los territorio rurales en la actualidad, los habitantes se caracterizan por poseer condiciones de pobreza elevadas; malas prácticas en el uso y explotación de recursos naturales, poca valoración de "lo rural" y acceso a oportunidades; que limitan sus condiciones de vida; las cuales sumadas a la profundización de desigualdades socio-económicas, baja calidad de la infraestructura, inequidad en la propiedad de la tierra, migración urbano - rural, ambientes de clientelismo, corrupción y violencia, evidenciados en gran parte de estos territorios; se constituyen como el principal obstáculo para alcanzar el desarrollo rural del país (GAMARRA, 2009; SALGADO, 2014)

Dadas las condiciones de precariedad y atraso del campo colombiano, en los últimos años se han desarrollado un número importante de leyes que denotan esfuerzos importantes por mejorar el contexto rural, pero cuyos resultados han sido insuficientes; y en territorios en su mayoría rural, como el Departamento de Sucre, han dejado una secuela significativa, llevando a estancamientos en el crecimiento, denotados en los aportes del PIB nacional con cifras constantes menores al 3\% anual (GOBERNACIÓN DE SUCRE, 2016).

Estas situaciones de precariedad y crecimiento desacelerado de la economía del Departamento de Sucre, conjunto a las dinámicas nacionales alrededor de los acuerdos de paz suscritos recientemente, llevan a evaluar el aporte de las propuestas de reformas agrarias realizadas en estos acuerdos y las posibilidades que tiene el campesinado del Departamento para mejorar las condiciones de vida y el desarrollo del mismo.

El análisis de estas propuestas se establece en el marco del Acuerdo para la Terminación del Conflicto celebrado en La Habana Cuba, entre el Gobierno Nacional Colombiano y las Fuerzas Armadas Revolucionarias de Colombia, FARC - EP, tras un proceso de concertación de más de 4 años, en la que se expone una Reforma Rural Integral- RRI, cuyo objetivo es establecer las bases para la transformación estructural del campo, a través de la creación de condiciones de bienestar para la población rural y contribuyendo a la construcción de una paz estable y duradera para el país (ALTO COMISIONADO PARA LA PAZ, 2016).

Exactamente, una transformación estructural del campo, que adopte medidas para promover el uso adecuado de la tierra, de acuerdo con su vocación y estimule la formalización, restitución y distribución equitativa de la misma, garantizando el acceso progresivo a la propiedad rural de quienes habitan el campo y en particular a las mujeres rurales y la población más vulnerable, regularizando y democratizando la propiedad y promoviendo la desconcentración de la tierra, en cumplimiento de su función social (ALTO COMISIONADO PARA LA PAZ, 2016).

\section{Materiales y métodos}

El presente trabajo es de tipo descriptivo-documental y parte del análisis de la evolución normativa colombiana para el desarrollo rural; el diagnóstico del Departamento de Sucre y la propuesta de reforma rural integral propuesta en el Acuerdo para la Terminación del Conflicto celebrado en La Habana Cuba, entre el Gobierno Nacional Colombiano y las Fuerzas Armadas Revolucionarias de Colombia, FARC (BLAS, SANCHES, \& TUJAGUE, 2010; HERNÁNDEZ SAMPIERI, 2014).

\section{Resultados y discusión}

Evolución de la normatividad rural en Colombia. A lo largo del tiempo, los avances en materia de ruralidad en Colombia han sido palpables, a través de la implementación de normas que han regido y vigilado al campesinado colombiano para la consecución de un 
desarrollo rural más competitivo y vanguardista con el panorama latinoamericano.

No obstante, contrario a trabajar el desarrollo rural, como un proceso de transformación, integración y fortalecimiento de las actividades agropecuarias y no agrícolas bajo un manejo sustentable de los recursos para el bienestar de los habitantes (BANCO INTERAMERICANO DE DESARROLLO, 2016; COLPOS, 2016); en Colombia este proceso se ha basado en un conjunto de proyectos y medidas políticas compensatorias para la población campesina más pobre; movidas en los últimos años bajo las perspectivas del Plan Nacional de Desarrollo, el proyecto de ley de desarrollo rural, las posibles opciones derivadas del acuerdo de la Habana y la Ley de 1448 de Victimas y Restitución de Tierras (SALGADO, 2014).

Dentro de esta serie de proyectos y medidas compensatorios para el campesinado colombiano, se pueden destacar:

Ley 160 de 1994 : En la cual se estipula como deber del estado promover el acceso progresivo a la propiedad de la tierra de los trabajadores agrarios y a otros servicios públicos rurales, con el fin de mejorar el ingreso y la calidad de vida de la población campesina y se crea el Sistema Nacional de Reforma Agraria.

En la medida que promueve y consolida la paz, elimina y previene la inequitativa concentración de la propiedad rústica o su fraccionamiento antieconómico; apoya a hombres y mujeres del campo, de escasos recursos, en los procesos de adquisición de tierras; fomenta una adecuada explotación y la utilización social de las aguas y de las tierras rurales; acrece el volumen global de la producción agrícola, ganadera, forestal y acuícola, en armonía con el desarrollo de los otros sectores económicos; aumenta la productividad de las explotaciones y la eficiente comercialización de los productos agropecuarios y procurar que las aguas y tierras se utilicen de la manera que mejor convenga a su ubicación y características (Ministerio de Agricultura y Desarrollo Rural, 1994).

Decreto no. 1777 de 1996: Por el cual se reglamenta parcialmente el Capítulo XIII de la Ley 160 de 1994, determinando las zonas de reserva campesina, y las define como aquellas que tienen por objeto el fomento y estabilidad la economía campesina, hacia la superación de las causas de los conflictos sociales que las afecten y logrando condiciones para la consecución de la paz y la justicia social en las áreas respectivas (MINISTERIO DE AGRICULTURA Y DESARROLLO RURAL, 1996).

Ley 731 de 2002: cuyo objeto es mejorar la calidad de vida de las mujeres rurales, priorizando las de bajos recursos y consagrar medidas específicas encaminadas a acelerar la equidad entre el hombre y la mujer rural; a través de la Participación de estas en los fondos de financiamiento del sector rural y la consideración de las mismas en la Reforma agraria (MINISTERIO DE AGRICULTURA Y DESARROLLO RURAL, 2002).

Ley 1448 de Junio de 2011- Ley de Víctimas y Restitución de Tierras: por la cual se dictan medidas de atención, asistencia y reparación integral a las víctimas del conflicto armado interno y se dictan otras disposiciones para los objetivos del ordenamiento agrario.

Incoder Resolución Número 1133: por la cual se fijan los patrones constitutivos de Unidades Agrícolas Familiares mínima a nivel predial, para los fines propios de la Convocatoria de Incentivo de Asistencia Técnica Rural. (INCODER, 2013).

El campo colombiano: un camino hacia el bienestar y la paz misión para la transformación del campo: este informe del Departamento Nacional de Planeación donde se establece la necesidad de colocar la equidad en el centro de las políticas de desarrollo del campo y la reducción de las desigualdades entre los habitantes rurales y urbanos, grupos étnicos y entre las distintas regiones para la generación de desarrollo. Señalando el requerimiento de políticas de Estado que eliminen las diferentes formas de exclusión social, el ordenamiento de la propiedad, estrategias para reducir la generalizada informalidad, la alta concentración en la estructura de la propiedad agraria y la situación de minifundio que viven estas zonas.

De igual forma, este informe muestra como objetivo de la Misión para la Transformación del Campo, el establecimiento de políticas de Estado para que la sociedad rural pueda manifestar todo su potencial, contribuyendo al bienestar nacional y haciendo un aporte decisivo a la construcción de la paz; una paz que ofrece, además, inmensas posibilidades para el desarrollo rural, tanto agropecuario como de la nueva ruralidad, y permite pensar en el avance de las zonas rurales como unos de los pilares del desarrollo futuro del país (DNP, 2015).

Plan Nacional de desarrollo, Todos por un nuevo país: mediante el cual se propone como enfoque fundamental de la política publica el bienestar de las comunidades rurales, a través de estrategias como el desarrollo rural integral que busquen corregir los desequilibrios regionales en la provisión de bienes públicos y servicios sociales, promover la igualdad de oportunidades para los pobladores rurales y el aumento significativo de la competitividad sectorial sobre la base de la agricultura familiar como principal fuente de 
ingresos en las áreas rurales, especialmente en territorios con alto riesgo de conflictividad social y económica. (PLAN NACIONAL DE DESARROLLO, 2016).

Diagnóstico del Departamento de Sucre para el emprendimiento de procesos locales hacia la superación de la pobreza, la violencia y el logro de la reconciliación y la paz.

El Departamento de Sucre, está ubicado al norte de Colombia, entre los $10^{\circ} 08^{\prime} 03^{\prime \prime}$ y $08^{\circ} 16^{\prime} 46^{\prime \prime}$ de latitud norte y los $74^{\circ} 32^{\prime} 35^{\prime \prime}$ y $75^{\circ} 42^{\prime} 25^{\prime \prime}$ de longitud oeste. Con una extensión territorial de 10.917 km²; bordada por la existencia de importantes recursos hídricos y forestales; que favorecen la formación de riquezas en el subsuelo y la vocación agropecuaria del Departamento (GOBERNACIÓN DE SUCRE, 2016).

A nivel demográfico, la población de Departamento, de acuerdo a proyecciones del DANE, es de 859.913 habitantes, en rango de edades jóvenes (52,9\% menores a 44 años), de los cuales el $49,3 \%$ son mujeres, y $44,9 \%$ habita en zonas rurales; la mayor parte de la población rural dispersa está en las edades de 5 a 19 años, pero también respecto al censo 2005 se evidencia un crecimiento de la población a partir de los 44 años y el ensanchamiento de la pirámide poblacional en cúspide lo cual refleja el fenómeno de transición demográfica hacia el envejecimiento. Este índice para Sucre, según el CNA 2014 es de 52,9\%, lo que significa que hay casi 53 adultos mayores por cada 100 menores de 15 años, reconoce el DANE que en el Censo Nacional de Población 2005, este índice estaba en 25 adultos; nótese como en 10 años se duplicó este indicador demográfico (Fig. 1).

Distribución (\%) de la población en el área rural dispersa censada según edad y sexo

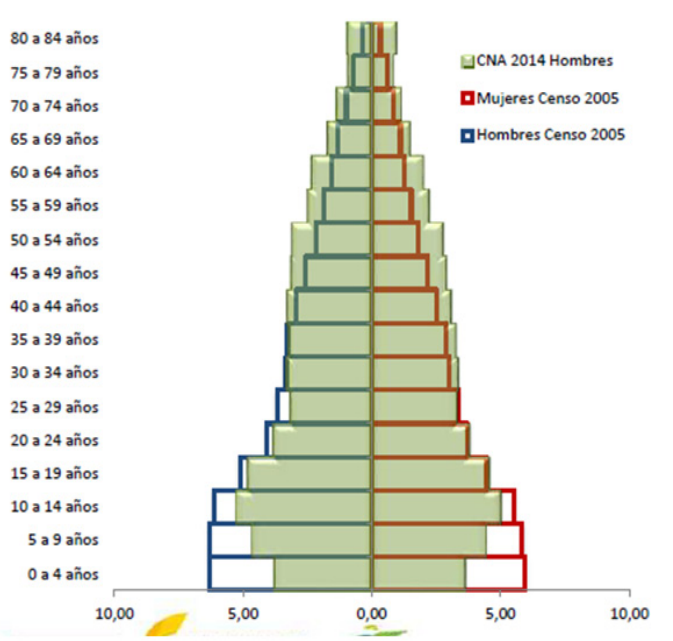

Figura 1. Pirámide poblacional área rural dispersa-Fuente: Tomado de DANE. CENSO NACIONALAGROPECUARIO (2014).
En relación a este contexto poblacional, al analizar la propuesta de reforma agraria integral, con el contexto demográfico del Departamento, se encuentran apartes que promueven la lgualdad y enfoque de género, expuestos en la propuesta de reforma rural integral:

"el reconocimiento de las mujeres como ciudadanas autónomas, sujetos de derechos que, independientemente de su estado civil, relación familiar comunitaria, tienen acceso en condiciones de igualdad con respecto a los hombres a la propiedad de la tierra y proyectos productivos, opciones de financiamiento, infraestructura, servicios técnicos y formación, entre otros; atendiendo las condiciones sociales e institucionales que han impedido a las mujeres acceder a activos productivos y bienes públicos y sociales." (ALTO COMISIONADO PARA LA PAZ, 2016).

Estas declaraciones denotan, intereses por el desarrollo de la población femenina de los territorios rurales, las cuales según datos de la CNA, son generalmente omitidas para la toma de decisiones de producción en las Unidades de Producción Agrícolas - UPA -extensión de tierra de $500 \mathrm{~m}^{2}$ o más (de acuerdo al territorio), dedicada total o parcialmente a la producción agropecuaria, considerada como una unidad económica - específicamente en el Departamento de Sucre, la participación en la toma de decisiones por parte de la mujer es tan solo el $28 \%$; lo cual, sumado al hecho de ser victimizadas tras hechos de conflicto y violencia en las últimas décadas en el Departamento, replantea la importancia del papel de la mujer campesina, en las consideraciones propuestas.

En relación a las condiciones de vida, Sucre es un Departamento con niveles de pobreza altos, de acuerdo a cifras del DNP el $44,7 \%$ de la población del Departamento es pobre por ingresos y según el CNA, el $59.5 \%$ de la población del área rural dispersa, hecho que se recrudece al mirar las cifras del índice de Necesidades Básicas Insatisfechas NBI, por zona, que reflejan disparidades al contar con el $46,60 \%$ en la cabecera y del $69,48 \%$ en el área rural, en estado de pobreza (GOBERNACIÓN DE SUCRE, 2016).

La economía del Departamento actualmente está concentrada en el sector terciario, que ha tomado relevancia en los últimos años a través de actividades como el comercio, la hotelería, servicios financieros, servicios sociales, entre otros; dejando a un lado la actividad del sector primario (AGUILERA, BERNAL, \& QUINTERO, 2006; GOBERNACIÓN DE SUCRE, 2016) (Fig. 2) 


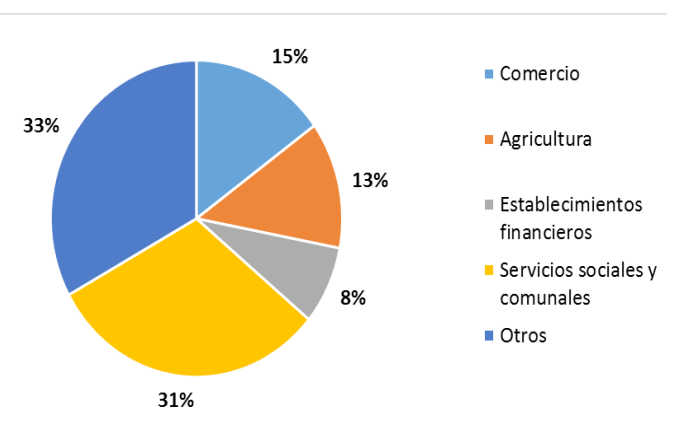

Figura 2. Principales áreas del PIB departamental 2015 Fuente: DNP -2016.

No obstante, a pesar de la tercerización de la economía, actividades alrededor de la agricultura, han mostrado aumentos en la participación del PIB en los últimos 4 años, con tasas de crecimiento de 1,5\%; que denotan la importancia del sector y refuerzan la consideración de éste como clave para el desarrollo del Departamento al concentrar gran parte de la vocación productiva de la población; donde las áreas rurales representan un $80,5 \%$, de las cuales, el $82 \%$ son Unidades de Producción Agropecuaria UPA y el 18\% producción no agropecuaria (DANE, 2015).

El uso de la tierra en el Departamento de Sucre se relaciona con su tenencia, del $1.428 .000 \mathrm{Ha}$. que cobijó el área rural dispersa censada en Sucre, el $64.5 \%$ corresponde a uso agropecuario y de esas 494,000 ha., el $82,6 \%$ está dedicada a pastos (425.000 ha.) y solo el $13,4 \%$ se dedica a uso agrícola (66.000 ha.). De la tierra destinada a uso agrícola. El $82,1 \%$ destina a cultivos transitorio, el $15,6 \%$ a permanentes y el $2,3 \%$ a asociados.

Según el Instituto Geográfico Agustín Codazzi IGAC, la tierra apta para actividades ganaderas y silvopastoriles asciende a 21,1 millones de hectáreas, y hoy se usan 39,2 millones (incluyendo malezas y rastrojos); lo que llevaría a considerar la liberación de al menos el 10,6\% del total del suelo utilizado para ganadería; Una cifra más realista indicaría que la tierra con vocación netamente pecuaria, que es de 15 millones, se compararía con las 31,6 millones utilizadas. Es decir, la liberación de tierras para agricultura y actividades forestales alcanzaría a cerca de 15 millones de hectáreas, ya que el uso en actividades silvopastoriles es prácticamente marginal.

Del total de áreas sembradas para uso agrícola, el 34\% está destinado al cultivo de cereales, el $29,4 \%$ a frutales y el 24,4 a plátanos y tubérculos. El $61,5 \%$ del total de área agrícola sembrada de cultivos agroindustriales corresponde a cultivos de café, palma y caña. (DANE, 2015) (Fig. 3).

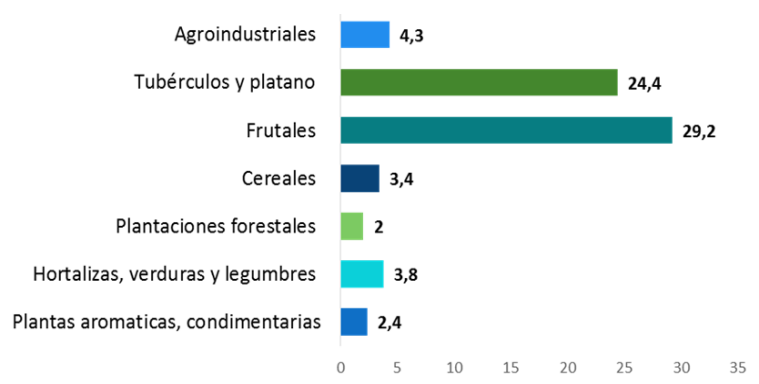

Figura 3. Usos área Cultivada. Fuente: DANE-2016.

En relación al uso de maquinaria para la producción agrícola el $92 \%$ de los campesinos manifestó no tener maquinaria, ni recibir asistencia técnica (91\%), el año anterior. Aquellos que acceden a asistencia técnica reconocieron participar de temáticas relacionadas a las buenas prácticas agrícolas, dictadas por el SENA (Tabla 1).

En relación a la producción, en el Departamento está dirigida principalmente para el autoconsumo $72,5 \%$ y se carece de apoyo técnico y financiero para las Unidades de Producción Agrícolas. Respecto al acceso al agua, en Sucre, según el censo, el 94,3\% de la población posee acceso y participación mayoritaria en más de una fuente de agua, pero su dificultad ésta en hacer uso, porque es escasa (81\%), sin embargo la protegen $(58,3 \%)$, conservan la vegetación $(58,1 \%)$ y planta arboles $(36,9 \%)$ que ayuda a su preservación. Esta práctica, el hecho de solo usar químicos en los cultivos, la reutilización de los desechos animales y

Tabla 1. Participación de la UPA en el área dispersa censada con tenencia o no.

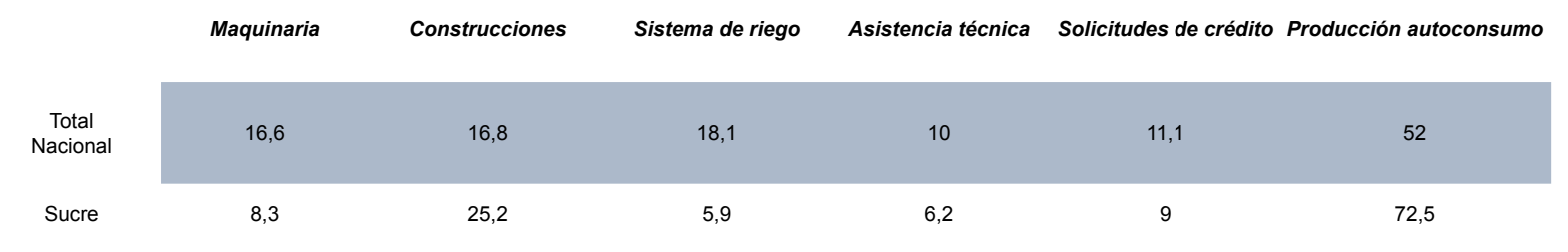


vegetales para utilizarlos en fertilización $(81,7 \%$,) el manejo y reutilización de desechos no orgánicos facilita el desarrollo sustentable del medio ambiente en el Departamento.

Con respecto a la vocación ganadera del Departamento, el $23 \%$ de las áreas rurales, cuenta con presencia de ganado bovino, y de otros tipo de especies como porcino, búfalos, equinos y caprinos (Tabla 2).

Tabla 2. Inventario pecuario y avícola. Área Rural dispersa.

$\begin{array}{cccc}\text { Ganado } & \text { Total nacional } & \text { Sucre } & \text { Participación \% } \\ \text { Bovino } & 21.400 & 820 & 3,80 \% \\ \text { Porcino } & 1.050 & 44 & 4,20 \% \\ \begin{array}{c}\text { Bufalino, equino, ovino y } \\ \text { caprino }\end{array} & 2.900 & 86 & 2,90 \% \\ \begin{array}{c}\text { Avícola } \\ \text { Fuente: DANE. CNA 2014. }\end{array} & 720.000 & 2.700 & 0,40 \%\end{array}$

A pesar de tener una pequeña participación en el agropecuario del país, la ganadería y la porcicultura son fuente importante de ingresos, pero el ganado Bovino, carece de especialización (carne o leche), tecnificación y por demás su modo de producción es extensiva.

De acuerdo con "anti ranking" de los Departamentos con los mayores conflictos de los suelos en Colombia 2014, del IGAG, Norte de Santander y Sucre son líderes de este escalafón, según el IGAG, Sucre cuenta con 1.072 .826 ha, de las cuales el $42 \%$ están sobre utilizadas y el $36 \%$ subutilizadas; lo que permite destacar el mal uso que los pobladores hacen del suelo, sin pensar en las consecuencias que puede representar para el Departamento en el mediano plazo, como el desabastecimiento de agua y la pérdida de áreas productivas que pueden afectar la seguridad alimentaria de la región.

En lo concerniente a la concentración de la tierra, al analizar la distribución de la tierra rural por tamaño de propiedad en el Departamento es posible determinar una fuerte concentración de tierras en pocos propietarios, dado que la mayoría posee pequeñas áreas, donde el uso y explotación es limitado; en detalle, el número de UPA de más de 500 ha. representan el $0,2 \%$ del total y ocupan el $17.5 \%$ del área rural dispersa, mientras que las UPA menores de 5 ha. representan el 67,3\% pero ocupan el $7,3 \%$ del área encuestada. De 50 ha, a menos de 500 el $4,3 \%$ del total y ocupan el $40 \%$ del total de área rural dispersa (Figura 4).

Todo lo anterior, en contraposición con los acuerdos de La Habana, nos conducen a pensar en la tenencia de tierras como un problema para el desarrollo del Departamento, dado que está tenencia estaría aumentando las brechas de pobreza de sus pobladores y reafirmando lo expuesto en la propuesta de reforma integral donde se reconoce la necesidad de:
"La democratización del acceso y uso adecuado de la tierra: mecanismos y garantías que permitan que el mayor número posible de hombres y mujeres habitantes del campo sin tierra o con tierra insuficiente puedan acceder a ella y que incentiven el uso adecuado de la tierra con criterios de sostenibilidad ambiental, de vocación del suelo, de ordenamiento territorial y de participación de las comunidades"(ALTO COMISIONADO PARA LA PAZ, 2016).

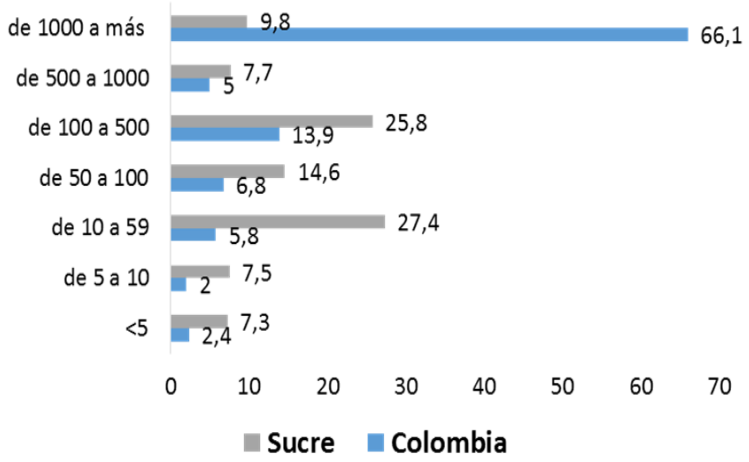

Figura 4. Distribución (\%) del área rural dispersa censada de las UPA por tamaño (ha) Fuente. DANE. Censo Nacional Agropecuario.

Perspectivas para el Departamento de Sucre a la luz de los acuerdos de La Habana y el censo rural 2014.

Al Analizar el contexto rural del Departamento de Sucre, e identificar las grandes brechas sociales y económicas en al que se encuentra; conjunto con la propuesta de reforma agraria del acuerdo de la Habana, uno de los puntos críticos que motivan este documento, es la tenencia de tierras; considerada como factor generador de conflicto, no solo en Colombia, si no a nivel mundial.

Específicamente para el Departamento de Sucre, el hecho de que el $4,5 \%$ de las unidades de producción agrícola representen la ocupación del $57.5 \%$ del área rural dispersa, y la agricultura esté perdiendo peso en la economía del Departamento; generando brechas y pobreza en la población campesina que la habita y sobrevive con una agricultura de pan coger, lleva a considerar como hecho económico fundamental, la propuesta de reforma agraria integral expuesta en los acuerdos de la Habana; dado que entre otros propone una redistribución de la tierra; donde los pobres y víctimas del conflicto tengan participación.

El hecho real según el Censo Agropecuario 2014, es que Las UPA en Sucre, de menos de 5 ha. representaron el $70,9 \%$ del total y ocuparon el $2,4 \%$ del área rural dispersa censada, dada la definición del Incoder para las UPAs en Sucre, la entrega de tierras para completar las extensiones que le hacen falta y la entrega de tierras 
a quienes no las tengan (victimas, desmovilizados, entre otros), del banco de tierras propuesto; ayudarán a resolver el problema del campesino sucreño.

Ahora bien, la redistribución no puede ir separada de la productividad agrícola; el desarrollo integral del campo, implica según el Acuerdo, el adecuado equilibrio entre la producción agrícola familiar, pero también agroindustria, agricultura comercial de escala, en otras palabras, en su implementación se considera producción a nivel industrial, ya sea por inversión privada o por mecanismos de asociación de campesinos con tierra, o una combinación de las dos formas de producción, revertir la producción agrícola para el autoconsumo, implica la transformación de los modos de producción del campo, situación también contempladas en los acuerdos, pero con una connotación especial, con encadenamientos verticales y horizontales en diferentes escalas.

La inversión privada y las políticas públicas de los entes territoriales, nacionales, departamentales y municipales deberán ir dirigidas al logro de estos objetivos, deberán impactar la economía rural del Departamento generando apoyo técnico y financiero para las Unidades de Producción Agrícola, para su producción, riego, y comercialización.

Las necesidades de mejorar las condiciones de vida del campesino sucreño que en la actualidad poseen un ingreso promedio de $\$ 440.000$ (Documento tres del Observatorio del Mercado de Trabajo en Sucre, 2014) y se mantiene en líneas de pobreza, hace latente la oportunidad que posee el Departamento ante una reforma rural integral; que lleve a un nuevo modelo de desarrollo económico que permita promover el desarrollo humano, visibilizar las diferencias de género y disminuir la discriminación, promover la sostenibilidad, generar democracia, confianza institucional y disminuir los conflictos.

Separarnos del sesgo al que ha llevado la preservación del orden social rural en el país, con todas sus inequidades y ataduras al desarrollo, fundamentadas en una estructura de la tenencia de la tierra convertida en inamovible para la política pública, jerarquización social, hegemonía política tradicional que restringe las elecciones libres, limpias y competitivas y que no hace un reconocimiento político del campesinado (PNUD, 2011); hacen necesario la generación de un cambio estructural para el Departamento.

La reforma rural integral concibe el desarrollo rural en función del territorio, centra la atención en los pobladores más excluidos y vulnerados y exige cambios en el modelo económico. Es categórica en rescatar el liderazgo sustantivo del Estado y en advertir que no es posible si no se erradican de raíz factores estructurales como la concentración de la propiedad rural, la pobreza, la miseria, y un orden social político injusto y excluyente. (ALTO COMISIONADO PARA LA PAZ, 2016)

Conclusiónes

El problema agrario es el resultado de una inequitativa distribución de la tierra, un modo de producción que privilegia la posesión y no la explotación, una miopía empresarial de los poseedores de la tierra, agravada por el conflicto y el narcotráfico, que ha sumido a los campesinos sucreños en condiciones de pobreza y exclusión; generando mayores brechas entre áreas urbanas y rurales y segregando a su población.

El establecimiento de un acuerdo de paz, ha acelerado el pago la deuda histórica que tiene el Estado y la necesidad de establecer una salida política con el principal grupo guerrillero que tiene Colombia; que de forma indirecta y considerando los posibles beneficios que traería su ejecución facilitarían el desarrollo del Departamento de Sucre, a través de la oportunidad que tendrían los desplazados y miles de víctimas, de recibir nuevamente tierra, asistencia técnica y financiera en el Departamento, como lo contempla el acuerdo. En gran parte resolvería el problema urbano relacionado con asentamientos en condiciones subnormales, la invasión de espacio público, informalidad en el empleo y la revictimizacióna la que se ha sometido esa población.

El acuerdo puede ayudar a resolver el problema de envejecimiento de la población rural y la incorporación de la generación campesina joven a las labores agropecuarias, claro con tierra, asistencia técnica, financiación y la modernización de los procesos de producción.

Convertir las UPAs en granjas inteligentes, explotadas con técnica y responsables con el medio ambiente, mejoraría la producción agropecuarias y por ende la reactivación económica del campo, retendría la población joven el área rural y seguramente impactaría en la calidad de vida de la población social, que no solo resolvería la seguridad alimentaria, si no que integraríamos a las unidades productoras campesinas a las cadenas productivas y a la comercialización que genere excedentes que permitan la acumulación y crecimiento.

Las Zonas de Reserva Campesina y las Zonas de Desarrollo Empresarial (Ley 160 de 2004), deben ser impulsadas nuevamente, con voluntad política del Estado, para convertirse en modelos de producción asociativos y zonas de desarrollo regional.

El reto de la sociedad es implementar, en este caso el acuerdo sobre lo rural, para consolidad la paz y construir un futuro libre de pobreza y exclusión para la población campesina del país. 


\section{Referencias}

AGUILERA, M., BERNAL, C., \& QUINTERO, P. (2006). Turismo y Desarrollo en el Caribe Colombiano. Cartagena: Documentos de trabajo en Economía Regional. Disponible en: http://www.banrep.gov.co/docum/Lectura finanzas/ pdf/DTSER-79.pdf. Consultado: 20-02-2017.

ALTO COMISIONADO PARA LA PAZ. (30 de 10 de 2016). Hacia un Nuevo Campo Colombiano: Reforma Rural Integral. Disponible en: http://www.altocomisionadoparalapaz.gov.co/herramientas/Documents/Acuerdo-Final-PuntoAgrario.pdf. Consultado: 23-01-2017.

ASHLEY, C., \& MAXWELL, S. (2001). Rethinking rural Development. Development Policy Review 1:395-425.

BANCO INTERAMERICANO DE DESARROLLO. (21 de 11 de 2016). Política de desarrollo rural. Disponible en: http://www.iadb.org/es/acerca-del-bid/politica-de-desarrollo-rural,6229.html. Consultado: 10-01-2017.

BANCO MUNDIAL. 2017. Datos Banco Mundial. Disponible en: http://datos.bancomundial.org/tema/ pobreza?view=chart. Consultado: 080-12-2016.

BLAS, H.; SÁNCHEZ, M.; TUJAGUE, M. 2010. El análisis descriptivo como recurso necesario en ciencias sociales y humanas. Fundamentos en humanidades, II.

BONET, J. 2006. Desequilibrios regionales en la política de descentralización en Colombia. Documentos de Trabajo sobre Economía regional. Banco de la República. Cartagena, Colombia.

COLPOS. (1 de 11 de 2016). Desarrollo rural sostenible. Obtenido de Colegio Mayor de posgrados. Disponible en: http://www.colpos.mx/wb pdf/Investigacion/LPI/lpi-10/PE\%20LPI\%2010.pdf. Consultado: 08-01-2017.

DANE. 2015. Censo agropecuario. DANE. Bogotá, Colombia.

DNP. 2015. El campo colombiano: un camino hacia el bienestar y la paz misión para la transformación del campo. DNP. Bogotá, Colombia.

GAMARRA, J. 2005. Desfalcos y regiones: un análisis de los procesos de responsabilidad fiscal en Colombia. Documentos de Trabajo sobre Economía regional. Banco de la República. Cartagena, Colombia.

GAMARRA, J. 2007. Pobreza Rural y Transferencia Tecnológica en la Costa Caribe. Documentos de Trabajo sobre Economía regional. Banco de la República. Cartagena, Colombia.

GAMARRA, J. R. 2009. Pobreza rural y transferencia de tecnología. Documentos de Trabajo sobre Economía regional. Banco de la República. Cartagena, Colombia.

GOBERNACIÓN DE SUCRE. 2016. Sucre progresa en Paz -Plan de desarrollo 2016-2016. Sincelejo.

HERNÁNDEZ SAMPIERI, R. 2014. Metodología de la Investigación. McGraw-Hill., Ciudad de México, México.

IICA. 2003. El enfoque territorial del desarrollo rural. Instituto Interamericano de Cooperación para la Agricultura. San José de Costa Rica. Costa Rica.

INCODER. 2013. Resolución número 1133 (2I Jun 2013). Ministerio de Agricultura y Desarrollo Rural Instituto Colombiano de Desarrollo Rural. Bogotá, Colombia.

JOSÉ, G. (s.f.). 1994. Pobreza rural y transferencia tecnológica en la Costa Caribe. Ministerio de Agricultura y Desarrollo Rural. Colombia.

LEY 160 DE 1994. Bogotá: Republica de Colombia. Disponible en: http://www.incoder.gov.co/documentos/ Ley160 2004.pdf. Consultado: 29-11-2016. 
MINISTERIO DE AGRICULTURA Y DESARROLLO RURAL. 1996. Decreto 1777 de 1996. Disponible em: www. desarrolloeconomico.gov.co/.../19-desarrollo-sostenible?...decreto-1777-1996. Consultado: 29-11-2016.

MINISTERIO DE AGRICULTURA Y DESARROLLO RURAL 2002. Ley 731 de 2002. Disponible en: https://www. minagricultura.gov.co/Normatividad/Leyes/Ley\%20731\%202002.pdf. Consultado: Consultado: 29-11-2016.

PLAN NACIONAL DE DESARROLLO. 2016. Todos por un nuevo país. Bogotá, Colombia.

PNUD. 2011. Informe de las Naciones Unidas: Colombia rural, razones para la esperanza. Bogotá, Colombia.

SALGADO, C. 2014. Colombia, estado actual del debate sobre el desarrollo rural. Desde Abajo Ed. Bogotá, Colombia. 